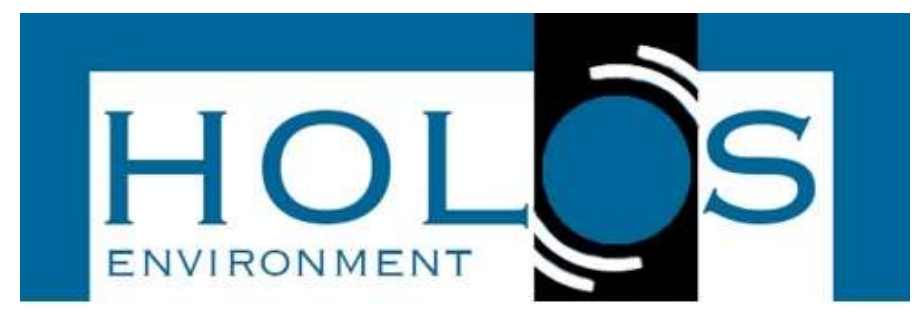

\title{
CONDUTIVIDADE HIDRÁULICA DA FORMAC̣ÃO RIO CLARO A PARTIR DE ENSAIOS GRANULOMÉTRICOS
}

\section{HYDRAULIC CONDUCTIVITY OF RIO CLARO FORMATION USING GRAIN SIZE ANALYSIS}

\author{
Roger Dias Gonçalves ${ }^{1}$, Hung Kiang Chang ${ }^{1}$
}

Artigo recebido em: 27/11/2017 e aceito para publicação em: 22/02/2018.

DOI: http://dx.doi.org/10.14295/holos.v18i1.12249

Resumo: A condutividade hidráulica $(K)$ é um fator determinante em relação às características de um aquífero, permitindo (ou não) a transmissão de fluidos e influenciando, assim, na velocidade de fluxo em diferentes camadas geológicas. Dessa forma, constitui fator-chave na compreensão do fluxo de água e de contaminantes. Neste trabalho, a granulometria do Aquífero Rio Claro é utilizada para mais bem entender a distribuição de suas propriedades hidráulicas, em particular da condutividade hidráulica, obtida pelos métodos de Hazen, Shepherd e Krumbein \& Monk. Os resultados foram comparados a testes de slug (Hvorslev) efetuados em poços existentes que exploram o aquífero. Verificou-se que a Formação Rio Claro apresenta grande variabilidade na relação areia/argila, sendo constituída por areias limpas, areias argilosas, até lentes silto-argilosas. A variabilidade granulométrica é grande próximo ao contato com a Formação Corumbataí, com mudanças marcantes nas quantidades de argila, silte e areia fina, além de contribuição expressiva de grânulos. Essa variabilidade litológica influencia na distribuição da condutividade hidráulica, cujos valores variam entre $8 \times 10-3 \mathrm{~cm} / \mathrm{s}$ e $2 \times 10-3 \mathrm{~cm} / \mathrm{s}$ em arenitos e são cerca de duas a três ordens de grandeza menores em lamitos. Os arenitos da Formação Rio Claro, apesar de espessuras restritas, possuem alta condutividade hidráulica, constituindo um ótimo reservatório de águas subterrâneas; porém, a presença de espessas lentes de argila pode atuar como barreiras hidráulicas. Desse modo, esta unidade aquífera tem o seu potencial de explotação subordinado à presença e continuidade espacial de camadas de arenitos com pouca matriz.

Palavras-chave: Condutividade Hidráulica. Aquífero Rio Claro. Granulometria de sedimentos. Testes de slug. Método de Shepherd.

Abstract: The hydraulic conductivity $(\mathrm{K})$ is a key hydraulic parameter of aquifers, conditioning the fluid transmission and governing groundwater velocity in distinct geological layers. Thus, a fundamental parameter for understanding groundwater and contaminant fluxes. In the present study grain size analysis was used to determine the hydraulic conductivity distribution of the Rio Claro Aquifer, based solely on the sediment physical properties. The K values were obtained by three methods - Hazen, Shepherd and Krumbein \& Monk - and the results were compared with slug tests (Hvorslev) carried out in existing wells. Rio Claro Formation presents a large range in sand/clay ratio, ranging from clean sands to silt and clay rich lenses. A large grain size distribution is observed near the contact with the Corumbataí Formation, ranging from clay, silt, fine sands to conglomerates. This lithological variability affects the $\mathrm{K}$ values distribution, with values ranging from $8 \times 10-3$ to $2 \times 10-3 \mathrm{~cm} / \mathrm{s}$ for sandstones and those for clay lenses of about two-three orders of magnitude lower. The Rio Claro Formation sandstones presents high hydraulic conductivity values, but the occurrence of thick clay lenses may act as hydraulic barriers. This aquifer unit is of limited thickness and presents high lithological heterogeneity, thus its productivity is highly dependent on the occurrence and spatial continuity of clean sandstone layers.

Keywords: Hydraulic conductivity. Rio Claro Aquifer. Grain Size Analysis. Slug test. Shepherd method

\footnotetext{
${ }^{1}$ Universidade Estadual Paulista (Unesp), Instituto de Geociências e Ciências Exatas, Rio Claro. Emails: (rdgon@hotmail.com, chang@rc.unesp.br)
} 


\section{INTRODUÇÃO}

O conhecimento sobre a distribuição das propriedades hidráulicas de um aquífero é de fundamental importância para a caracterização do fluxo de águas subterrâneas e, consequentemente, para o desenvolvimento de modelos (conceituais e numéricos) que descrevam este fluxo e forneçam subsídios para tomadas de decisão com relação a esses recursos. A condutividade hidráulica $(K)$ da porção saturada figura entre as propriedades mais importantes na avaliação do meio físico subterrâneo, sendo fundamental no estudo de transporte de contaminantes, análise de vulnerabilidade, velocidade de fluxo de águas subterrâneas, avaliação de métodos de remediação, entre outros (CHAPUIS et al., 2005; CHEONG et al., 2008; ROSAS et al., 2014; GONÇALVES e CHANG, 2017). Para a determinação dos valores de K são necessários ensaios que podem ser realizados em campo ou laboratório. Diversos métodos podem ser empregados, como uso de permeâmetros de carga constante e variável em laboratório, testes de bombeamento, traçadores ou slug tests executados in situ, e ainda métodos indiretos que se utilizam de modelos empíricos a partir de propriedades da rocha/solo (TERAMOTO et al., 2010). Todos os métodos possuem incertezas e alguma limitação, mas quando bem executados e os resultados devidamente avaliados são úteis para orientar as tomadas de decisão.

Os arenitos cenozoicos da Formação Rio Claro, arcabouço do aquífero homônimo, encontram-se sobrepostos aos siltitos da Formação Corumbataí na região de Rio Claro (SP). A Formação Rio Claro é composta predominantemente por arenitos e lamitos. Os arenitos variam de finos a grossos, por vezes conglomeráticos, pouco a muito argilosos (FERREIRA, 2005). A deposição da unidade é atribuída a ambiente fluvial meandrante por diversos autores, como Melo et al. (1997), Ferreira e Caetano-Chang (2008) e Teramoto et al. (2010). A espessura da unidade é variável, podendo chegar a 30 metros.

A Formação Corumbataí é a unidade superior do Grupo Passa Dois (PermoCarbonífero) na região e é constituída predominantemente por siltitos e argilitos cinzaavermelhados e arroxeados, com ocorrências localizadas de lentes de arenitos muito finos (COTTAS, 1983). Esta unidade é muito importante para o polo cerâmico regional (PAULA E SILVA e CHANG, 2017), visto que os argilitos constituem o insumo básico para a produção de produtos cerâmicos, e atua regionalmente como aquitardo. Quanto à 
Formação Rio Claro, além dos pacotes arenosos, a água subterrânea aí armazenada é largamente explorada.

Segundo Stradioto e Chang (2010), o Aquífero Rio Claro é raso e do tipo livre; suas águas apresentam características de águas pluviais de boa qualidade, armazenadas em reservatórios constituídos por litotipos predominantemente arenosos com elevado potencial de extração.

Com o objetivo de avaliar as propriedades hidráulicas do Aquífero Rio Claro, mais especificamente sua condutividade hidráulica, procedeu-se a um estudo detalhado da granulometria de sedimentos da Formação Rio Claro na região do município homônimo. A área estudada corresponde ao município de Rio Claro, localizado no centro-leste do estado de São Paulo. A figura 1 mostra a localização dos poços e afloramentos estudados, com ênfase para o Câmpus da UNESP em Rio Claro, localizado na porção sudeste do município, e adjacências. 
Figura 1 - Mapa de localização da área de estudo. Destaque para a área do Câmpus UNESP em Rio Claro

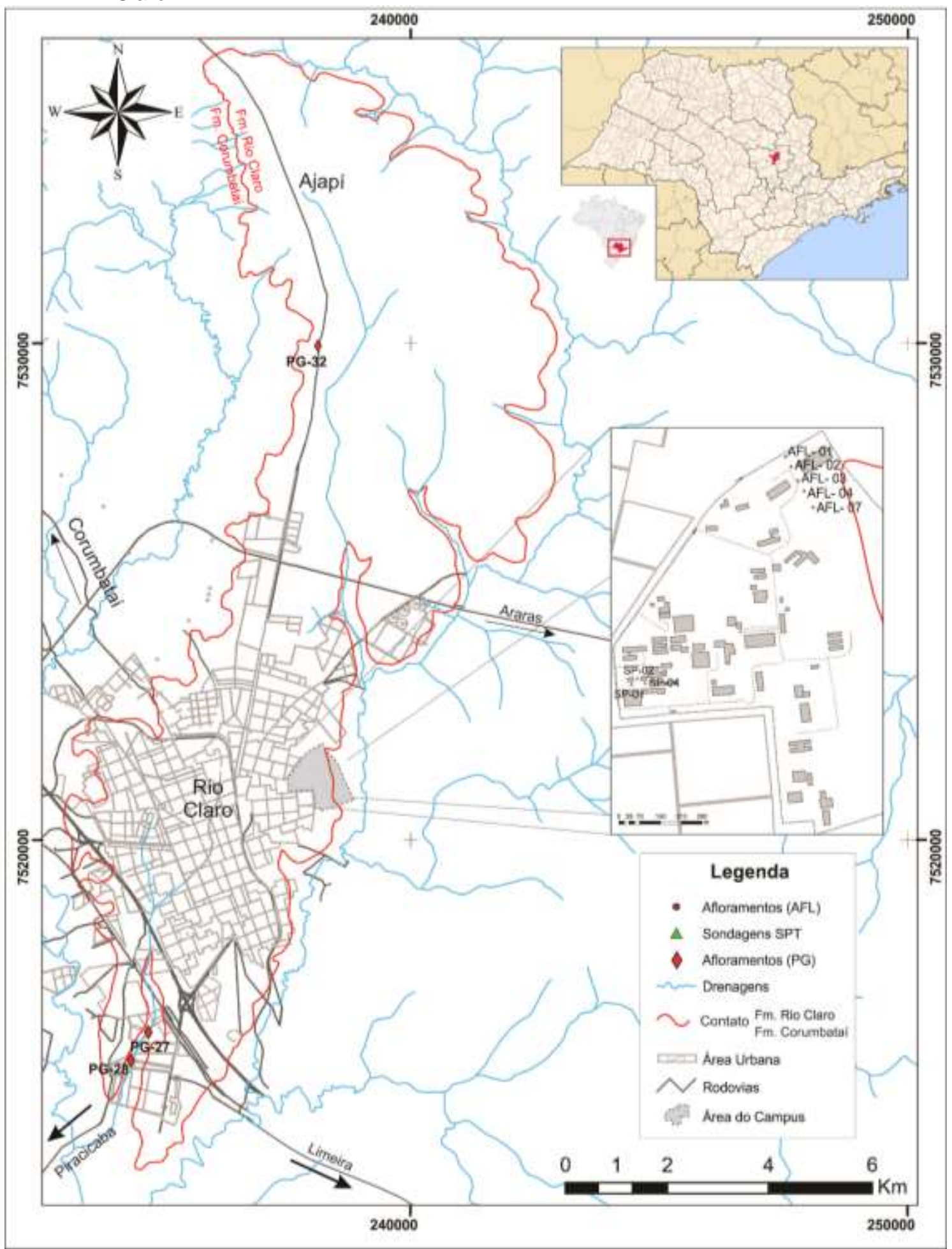

\section{MATERIAL E MÉTODOS}

Foram utilizadas amostras recuperadas de sondagens e coletadas em afloramentos das formações Rio Claro e Corumbataí, envolvendo etapas de campo e laboratório. As 
propriedades físicas dos materiais foram determinadas por meio de ensaios comumente utilizados em investigações geotécnicas, tais como granulometria conjunta e massa específica dos sólidos, conduzidos segundo as normas ABNT-NBR:7181 (ABNT, 1984) e ASTM D854 (ASTM, 1998). Foram efetuadas análises granulométricas utilizando os métodos de peneiramento e sedimentação, este último baseado na Lei de Stokes, com vistas a abarcar toda a gama de granulometrias que compõem as unidades de interesse. Como limite entre as frações grossa e fina utilizou-se a granulação de 0,062 mm, correspondente ao limite entre areia muito fina e silte na classificação de Wentworth. É possível observar em curvas cumulativas uma quebra no ponto de junção entre os diferentes métodos, e qualquer feição incomum neste ponto pode ser considerada como incerteza experimental.

A partir dos dados obtidos, a condutividade hidráulica foi determinada por meio da distribuição granulométrica dos sedimentos utilizando-se os métodos de Hazen (1911; in FETTER, 2001), Shepherd (1989) e Krumbein \& Monk (1942). O método de Hazen é efetivo em areias em que o diâmetro efetivo do grão $\left(d_{10}\right)$ situa-se aproximadamente entre $0,1 \mathrm{~mm}$ e 3,0 mm. Na fórmula aplicada ao método de Hazen (Equação 1): K é a condutividade hidráulica $(\mathrm{cm} / \mathrm{s})$; d 10 é o diâmetro efetivo do grão $(\mathrm{cm})$; C é um valor adimensional que pode ser obtido experimentalmente (areias finas, como as da Formação Rio Claro, possuem um valor aproximado de 60).

$$
K=C\left(d_{10}\right)^{2}
$$

O método de Shepherd considera que a condutividade hidráulica está relacionada ao diâmetro e à maturidade dos grãos, sendo calculada pela Equação 2, onde: $\mathrm{C}$ é o fator de ajuste, obtido experimentalmente; $d_{50}$ é o diâmetro efetivo do grão $(\mathrm{mm})$; j é o expoente obtido experimentalmente que leva em consideração a textura dos sedimentos. $\mathrm{O}$ fator $\mathrm{C} \mathrm{e}$ o expoente j possuem valores altos para sedimentos maturos e decaem para sedimentos imaturos. Para a Formação Rio Claro utilizou-se $C=100 \mathrm{e} \mathrm{j}=1,5$, correspondente a sedimentos parcialmente consolidados.

$$
K=C d_{50}^{j}
$$

Foi testada também a fórmula de Krumbein e Monk (1942) para fins de comparação entre os métodos, já que, diferentemente dos métodos acima, esta equação utiliza a permeabilidade do sedimento no cálculo e foi testada em areias inconsolidadas, onde: $d_{w}-$ 
diâmetro médio dos grãos $(\mathrm{mm}) ; \sigma_{\Phi}$ - desvio padrão; $\mathrm{k}$ - permeabilidade em darcys. Este método é efetivo quando o diâmetro médio varia entre $0,42 \mathrm{~mm}$ e 1,682 $\mathrm{mm}$ e o desvio padrão situa-se na faixa entre $0,574 \mathrm{~mm}$ e $0,973 \mathrm{~mm}$ (USACE, 2005).

$$
k=760 d_{W}^{2} \exp \left(-1,31 \sigma_{\Phi}\right)
$$

Esses valores foram então comparados a dados estimados em trabalhos anteriores (OLIVA, 2006; OLIVA et al., 2005) e dados obtidos a partir de ensaios de permeabilidade realizados em poços de monitoramento localizados no Câmpus da UNESP. O ensaio utilizado para a determinação da condutividade hidráulica foi o teste de slug, realizado em nível variável (zona saturada) aplicando-se uma carga ou descarga com a introdução ou remoção súbita de um volume dentro do poço, geralmente um sólido cilíndrico de volume conhecido, de forma que o nível d'água (NA) fosse elevado ou rebaixado instantaneamente. O monitoramento da posição do NA resulta em uma curva de rebaixamento ou recuperação deste no tempo, a partir da qual são extraídos os parâmetros que, em conjunto com as características geométricas do poço, fornecem o valor de condutividade hidráulica. Para interpretação da curva NA versus tempo foi utilizado o método de Hvorslev (1951) (Equação 4), onde: $K$ é a condutividade hidráulica; $r_{c}$ é o raio do revestimento; $R$ é o raio do poço; $L_{e}$ é o comprimento do filtro saturado; $T_{0}$ é o tempo de resposta (37\% de recuperação).

$$
K=\frac{r_{c}^{2} \ln \left(L_{e} / R\right)}{2 L_{e} T_{0}}
$$

Para execução e interpretação dos ensaios utilizou-se o kit Winslug (CHANG e CARNIER NETO, 2015).

\section{RESULTADOS E DISCUSSÃO}

\subsection{Distribuição Granulométrica}

Para melhor entendimento da distribuição tridimensional dos litotipos pertencentes à Formação Rio Claro, foram amostrados testemunhos de três ensaios SPT (Standard Penetrating Test) realizados no Câmpus da UNESP, afloramentos (AFL) em um corte de aproximadamente 200 metros de comprimento por 6 metros de altura, no Fórum Criminal de Rio Claro, a nordeste do Câmpus, e afloramentos distribuídos na área (PG - Figura 1). A partir das análises realizadas em laboratório foram geradas curvas granulométricas das 
amostras coletadas. Esses resultados foram agrupados em cinco litofácies: (1) Formação Rio Claro - amostras com teor de lama (silte + argila) abaixo de 20\%; (2) Formação Rio Claro - amostras com teor de lama entre 20\% e 40\%; (3) Formação Rio Claro - amostras silto-argilosas; (4) amostras do contato da Formação Rio Claro com a Formação Corumbataí; e (5) amostras da Formação Corumbataí.

As curvas granulométricas de frequência acumulada das três litofácies da Formação Rio Claro mostraram-se unimodais (Figura 2), porém com modas distintas entre si, variando de: areia fina a areia média na fácies com até $20 \%$ de lamas (Figura 2a); silte a areia média nas amostras com lamas entre $20 \%$ e 40\% (Figura 2b), argila a areia média na fácies com percentual maior que $40 \%$ de lamas (Figura 2c). As lentes de lamitos da Formação Rio Claro apresentam cerca de $22,5 \%$ de areia fina a média (Figura 2c). As amostras da Formação Corumbataí também exibem curvas unimodais, com composição predominantemente lamítica e cerca de $5 \%$ de areia (Figura 3a). Já as amostras coletadas no contato entre as unidades exibem curvas bimodais, com patamares que indicam mudança de granulação brusca e descontínua (Figura 3b), refletindo níveis conglomeráticos e lentes centimétricas com grânulos e seixos dispersos da base da Formação Rio Claro. A variação granulométrica de argila a grânulo evidencia a heterogeneidade das amostras, constatando uma zona de transição.

O coeficiente de uniformidade (CU) é um parâmetro obtido através da curva granulométrica que indica a uniformidade da amostra; no entanto, em apenas três amostras se alcançou o Di $i_{10}$ necessário para o cálculo: GR-08 com CU=2,55; GR-17 com CU=3,0 e GR-24 (contato) com CU=1466,7. Tais resultados mostram que apenas as amostras GR08 e GR-17 podem ser consideradas uniformes, pois o coeficiente é menor ou igual a 3. Importante pontuar que a amostra GR-17 constitui uma lente de areia média bem selecionada presente a $16 \mathrm{~m}$ de profundidade, $1 \mathrm{~m}$ acima do contato entre as unidades litoestratigráficas, refletindo a variabilidade litológica da Formação Rio Claro. 
Figura 2 - Curvas de análise granulométrica conjunta das amostras da Formação Rio Claro com menos de $20 \%$ de lamas (a), entre $20 \%$ e $40 \%$ de lamas (b) e lentes silto-argilosas (c)

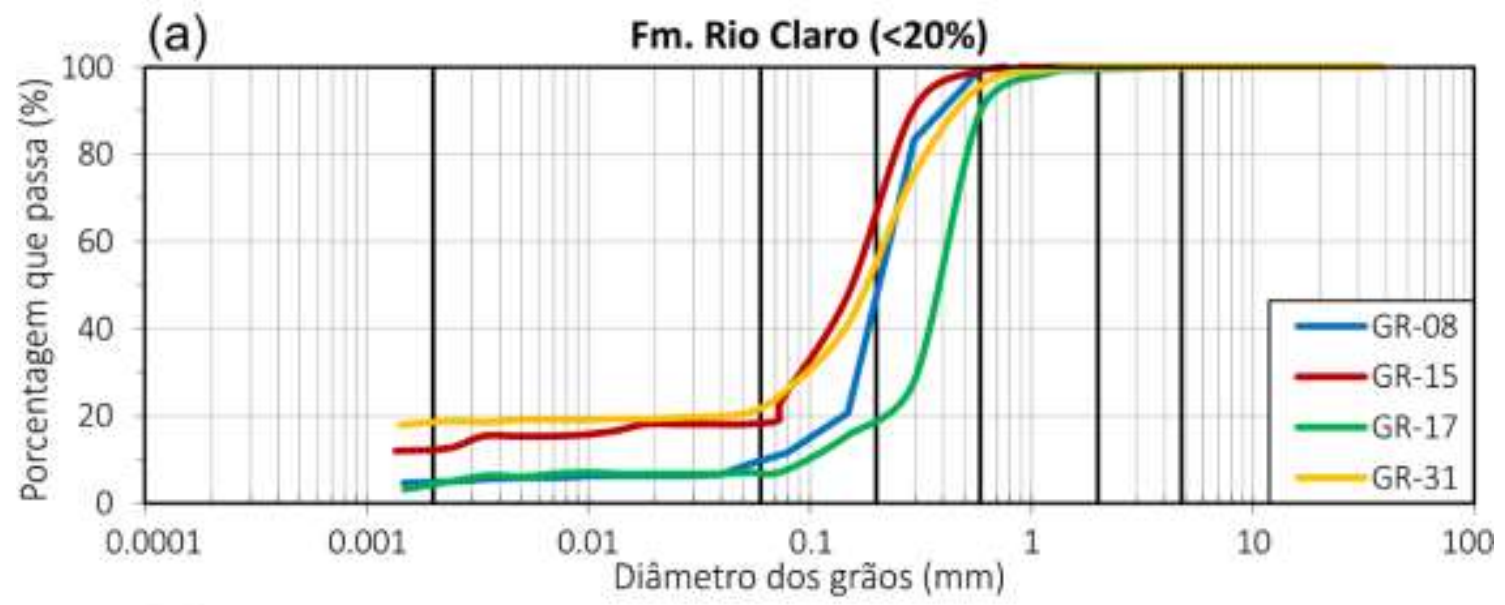

(b)

Fm. Rio Claro (20 - 40\%)

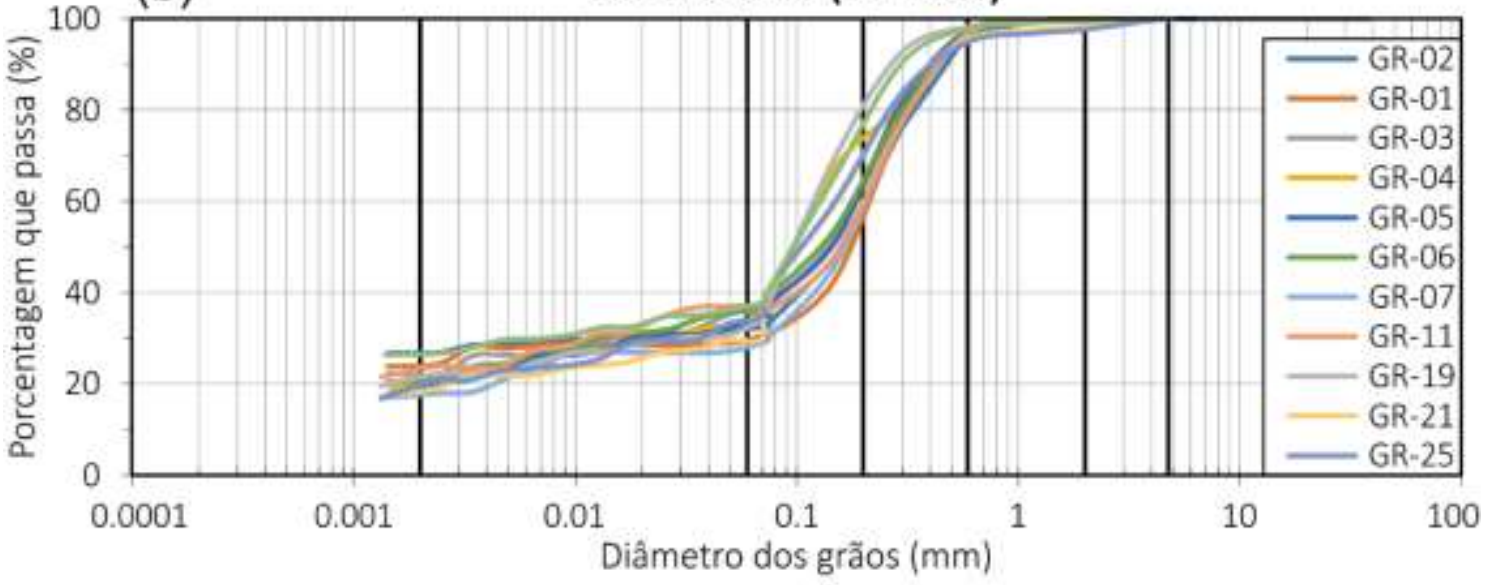

(c) Lentes de argila da Fm. Rio Claro (>40\%)

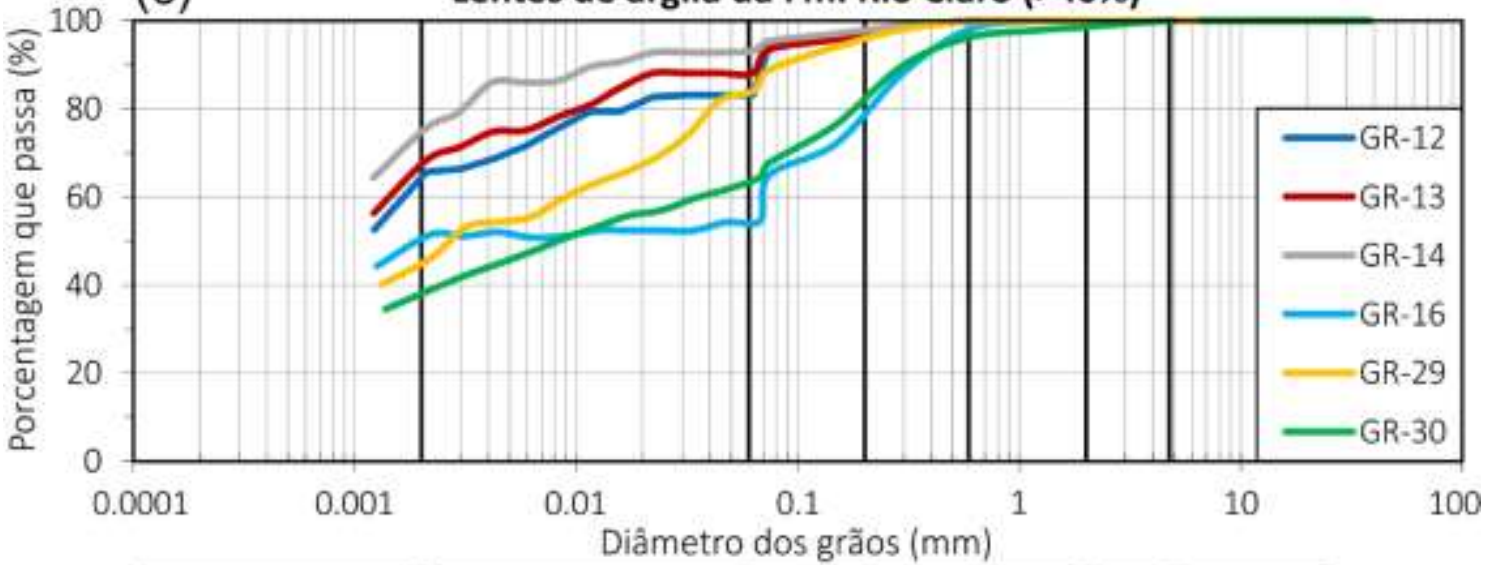

Argila

Silte

\begin{tabular}{|l|c|c|c|c|}
\hline \multicolumn{3}{|c|}{ Areia } & Grån & Seixo \\
\hline Fina & Média & Grossa & & S \\
\hline
\end{tabular}


Figura 3 - Curvas de análise granulométrica conjunta das amostras da Formação Corumbataí (a) (a) e amostras coletadas no contato Formação Rio Claro/Formação Corumbataí (b)

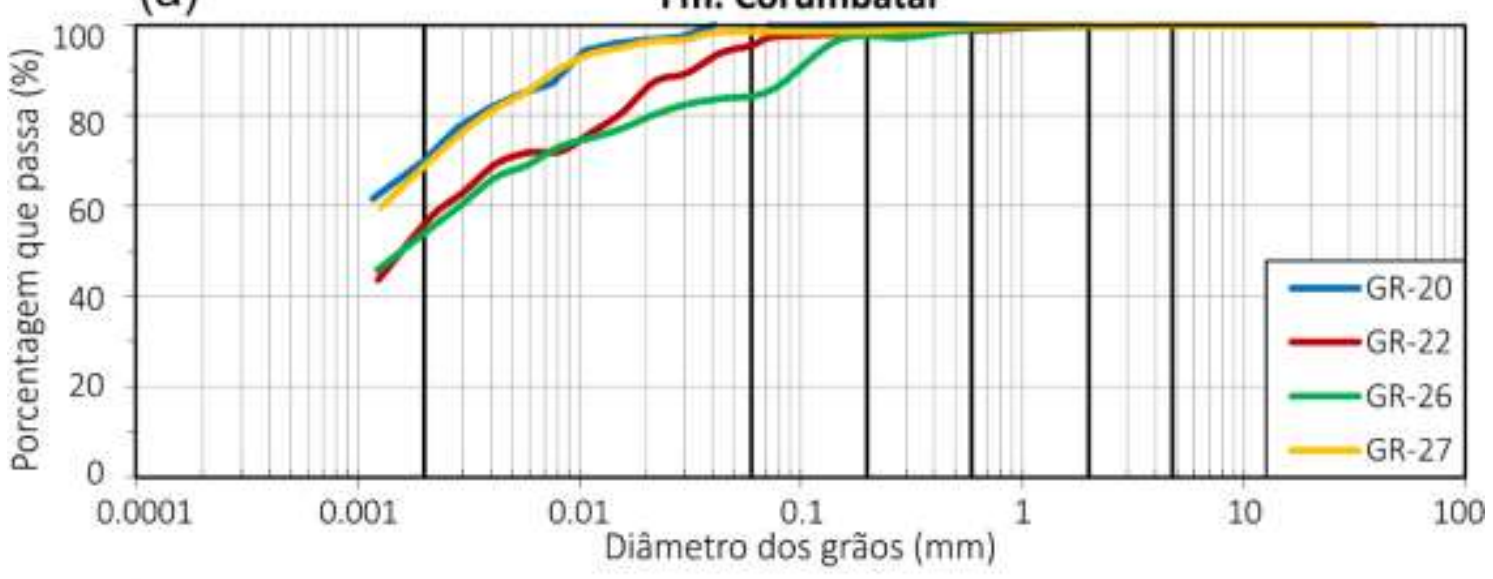

(b) Contato

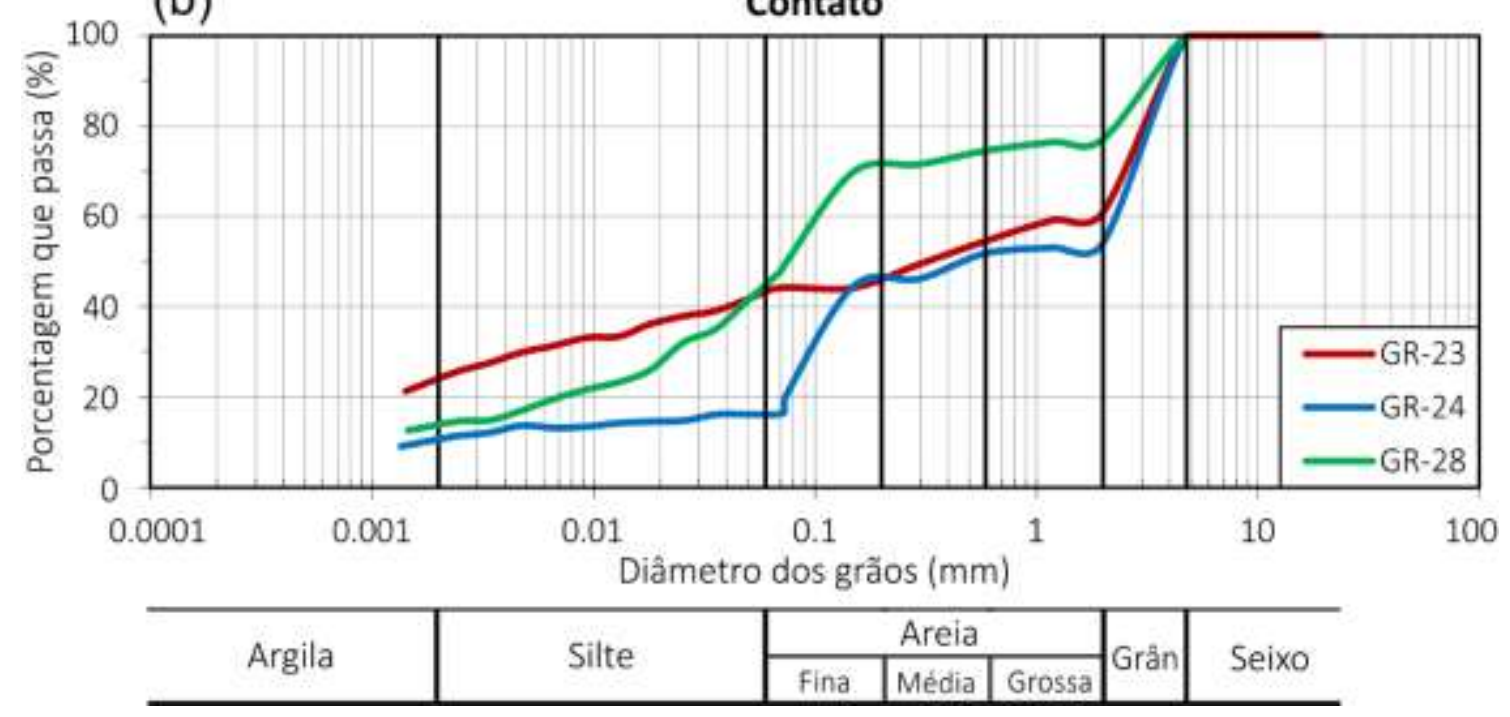

\subsection{Caracterização da Condutividade Hidráulica}

A partir das curvas granulométricas foram calculados os valores de condutividade hidráulica das cinco litofácies identificadas. O método mais eficiente para o cálculo das condutividades hidráulicas foi a equação de Shepherd, pois possibilitou o cálculo de K para a maioria das amostras, já que em apenas três delas obteve-se o parâmetro $\mathrm{Di}_{10}$ necessário para a equação de Hazen, e apenas duas amostras apresentaram o desvio padrão necessário para a equação de Krumbein \& Monk.

Os resultados de condutividade hidráulica obtidos por esses diferentes métodos mostram discrepância (Figura 4), em parte porque são utilizadas diferentes variáveis para cada equação. Enquanto o método de Hazen utiliza apenas o diâmetro efetivo, o método de Shepherd também utiliza as características texturais dos grãos; para o cálculo pelo método de Krumbein \& Monk, por sua vez, calcula-se a permeabilidade do sedimento a 
partir do diâmetro médio dos grãos e desvio padrão na escala lognormal e posteriormente sua transformação em condutividade hidráulica.

Figura 4 - Gráfico log de barras comparando os valores de condutividades hidráulicas calculadas por diferentes métodos

Comparação entre os Métodos
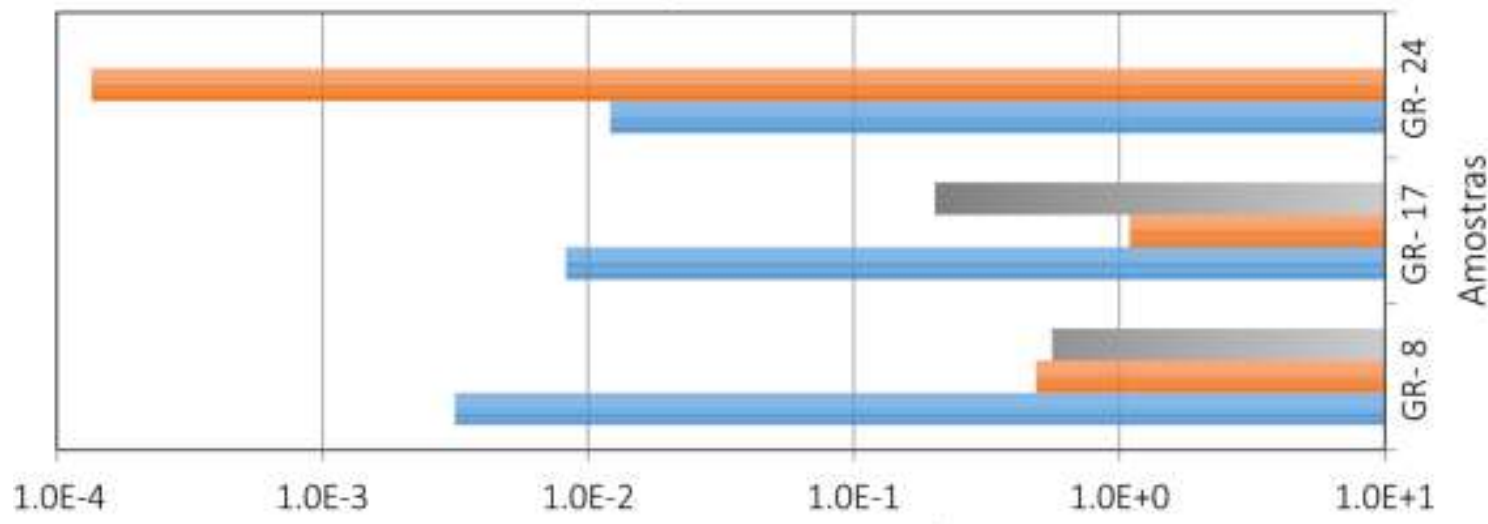

Condutividade Hidráulica $(\mathrm{cm} / \mathrm{s})$

= Método de Krumbein e Monk = Método de Hazen $\equiv$ Método de Shepherd

O gráfico da Figura 5 apresenta a variação da condutividade hidráulica calculada pelo método de Shepherd, enquanto os perfis litológicos revelam a distribuição granulométrica de acordo com a profundidade das sondagens. O método de Shepherd apresentou resultados consistentes com as amostras coletadas nas sondagens realizadas. Nos perfis de variação de condutividade hidráulica, as amostras coletadas até profundidades próximas de $8 \mathrm{~m}$ foram descritas como arenitos siltosos $(20 \%$ a $40 \%$ de lamas), sobrepostas a uma lente de lamito de cerca de $3 \mathrm{~m}$ de espessura. Abaixo da lente de lamitos mais rasa ocorre material arenoso, fino e de cor creme avermelhada de cerca de $3 \mathrm{~m}$ de espessura. Mais abaixo ocorre uma segunda lente de lamito, com aproximadamente $2 \mathrm{~m}$ de espessura, sobreposta a material arenoso, similar ao identificado acima. 
Figura 5 - Perfil de variação da condutividade hidráulica (pelo método Shepherd) das sondagens SP-01 e SP-04 e litologias correspondentes. Escala granulométrica: Amg (areia muito grossa); Ag (areia grossa); Am (areia média); Af (areia fina); Amf (areia muito fina); S (silte); e A (argila)

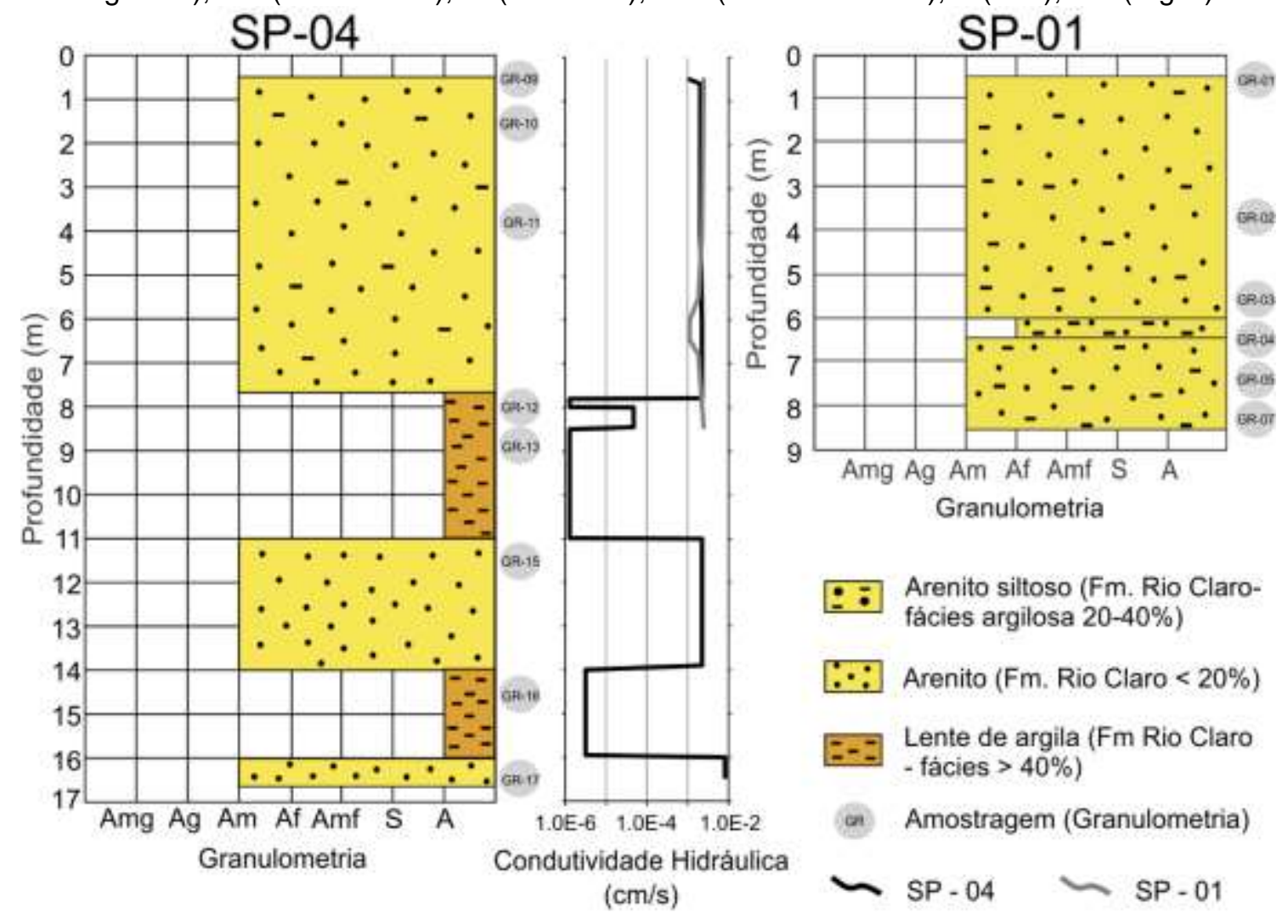

Quanto à Formação Rio Claro, os resultados mostram três fácies sedimentares que diferem principalmente pela porcentagem de argila e silte contida nas amostras. Na fácies com até $20 \%$ de lamas, a condutividade hidráulica calculada pelo método de Shepherd varia entre $2,2 \times 10^{-3} \mathrm{~cm} / \mathrm{s}$ e $8,3 \times 10^{-3} \mathrm{~cm} / \mathrm{s}$; na fácies com $20 \%$ a $40 \%$ de lamas, varia entre $1,2 \times 10^{-3} \mathrm{~cm} / \mathrm{s}$ e $2,5 \times 10^{-3} \mathrm{~cm} / \mathrm{s}$; e nas amostras com mais de $40 \%$ de lamas, varia entre $5,0 \times 10^{-6} \mathrm{~cm} / \mathrm{s}$ e $5,0 \times 10^{-5} \mathrm{~cm} / \mathrm{s}$. Nas amostras da Formação Corumbataí, os valores de condutividade hidráulica obtidos estão na faixa de $9,6 \times 10^{-7} \mathrm{~cm} / \mathrm{s}$ a $2,5 \times 10^{-6} \mathrm{~cm} / \mathrm{s}$; as condutividades hidráulicas dos sedimentos amostrados junto ao contato apresentam ampla variação, de $10^{-2} \mathrm{~cm} / \mathrm{s}$ a $10^{-4} \mathrm{~cm} / \mathrm{s}$. As curvas e parâmetros obtidos são compatíveis com o esperado das amostras, correspondendo às características inerentes a cada unidade.

A Figura 6 apresenta os valores de condutividade hidráulica estimados com base na distribuição granulométrica pelo método de Shepherd e com base em testes de slug pelo método de Hvorslev, além de resultados obtidos em trabalhos anteriores pelos mesmos métodos. Valores de K para a Formação Rio Claro, determinados por Oliva et al. (2005) e Oliva (2006) pelo método de Shepherd, quando comparados com os resultados por agrupamento deste estudo, evidenciam que os valores outliers presentes na Formação Rio 
Claro refletem a ampla variação decorrente da presença de lentes de lamitos e da heterogeneidade presente na base da unidade. Os valores médios de condutividade hidráulica estimados em testes de slug, tanto em estudos anteriores quanto os realizados neste trabalho, revelam boa correspondência com os valores calculados para as fácies arenosas da Formação Rio Claro, indicando que, apesar da presença de lentes de lamitos, são os pacotes arenosos os maiores responsáveis pelas boas características hidráulicas do aquífero. No entanto, verifica-se que os testes de slug apresentam uma variação significativa nas estimativas de condutividade hidráulica, o que pode ser interpretado como influência direta da presença de espessas lentes de lamito que ocorrem localmente nesta unidade geológica. Consequentemente, essas heterogeneidades, típicas de depósitos de ambiente fluvial meandrante, diminuem o potencial de explotação das águas subterrâneas do Aquífero Rio Claro. Neste cenário, apesar de elevados valores de condutividade hidráulica atribuídos a seus arenitos, este aquífero tem o seu potencial de explotação viabilizado apenas na presença e continuidade espacial de camadas de arenitos com poucos finos.

Figura 6 - Diagrama de caixa dos valores de condutividade hidráulica estimados por Shepherd, estimados em trabalhos anteriores (OLIVA, 2006; OLIVA et al. 2005) e resultados dos testes de slug

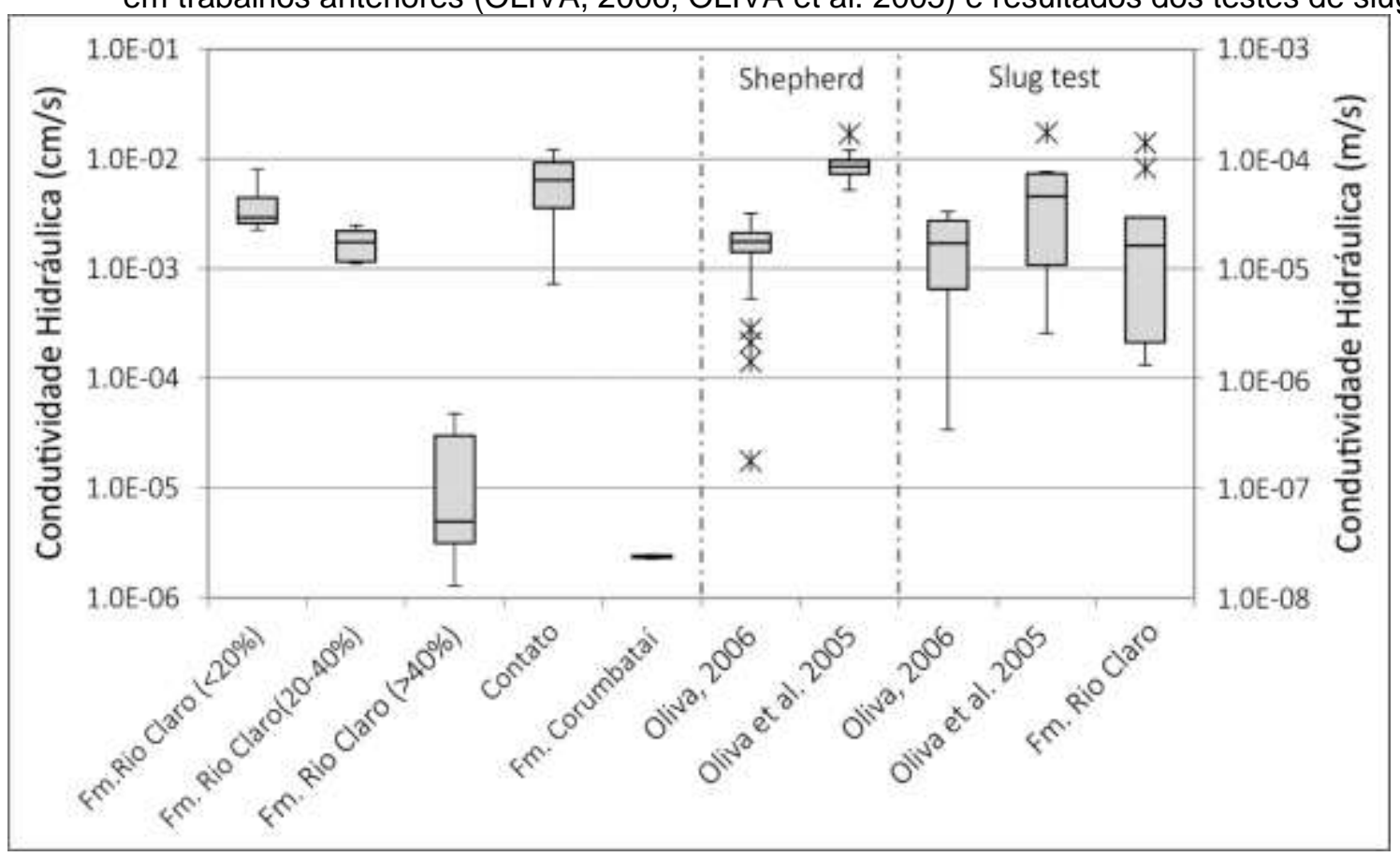




\section{CONCLUSÕES}

As curvas de distribuição granulométrica, geradas por ensaios de granulometria conjunta, evidenciam propriedades físicas das litofácies de modo simplificado, tornando possível a determinação da condutividade hidráulica dos sedimentos, que pode ser calculada a partir de diferentes métodos, como Shepherd e Hazen. Tais estimativas podem ser comparadas com aquelas provenientes de outros métodos, como testes de slug.

A Formação Rio Claro apresenta grande variabilidade da relação areia/argila, possuindo desde areias limpas até lentes ricas em silte e argila. A Formação Corumbataí, principal unidade sotoposta à Formação Rio Claro na área de estudo, apresenta prevalência das frações silte e argila, sendo que a areia fina contribui com um máximo de 15\% em sua composição. Em relação às amostras coletadas próximas ao contato entre as duas unidades, nota-se uma grande variabilidade granulométrica, com ampla variação nas quantidades de argila, silte e areia fina, com grande contribuição de grânulos (até 45\%) nessas amostras.

Por meio das análises granulométricas, a condutividade hidráulica dos arenitos da Formação Rio Claro foi estimada com mais precisão pelo método de Shepherd, já que o método de Hazen, além de necessitar diâmetros efetivos entre $0,1 \mathrm{~mm}$ e 3,0 mm que não foram encontrados nas amostras, demonstrou superestimar os valores de condutividade, fato este também observado por Oliva et al. (2005). Provavelmente isso ocorre porque o diâmetro utilizado é o efetivo, não levando em consideração características texturais e outros aspectos geométricos. As estimativas por Shepherd se aproximam dos dados obtidos pelos testes de slug realizados na área. Os valores médios obtidos por slug tests se encontram dentro do intervalo de condutividade hidráulica estimada por Shepherd para os arenitos da Formação Rio Claro, entre $8 \times 10^{-3} \mathrm{~cm} / \mathrm{s}$ e $2 \times 10^{-3} \mathrm{~cm} / \mathrm{s}$. Os baixos valores de $\mathrm{K}$ das lentes de lamito da unidade, cerca de duas a três ordens de grandeza menores, refletem-se em valores mais baixos observados nos testes de slug. Assim como a Formação Corumbataí atua regionalmente como uma base impermeável para o Aquífero Rio Claro, a ocorrência de espessas lentes de lamitos podem atuar como barreiras hidráulicas para o fluxo de águas subterrâneas nesta unidade.

Os arenitos da Formação Rio Claro possuem boa condutividade hidráulica, e tendo a Formação Corumbataí como uma base de baixa condutividade, constitui um ótimo armazenador para o Aquífero Rio Claro. No entanto, esta unidade aquífera é limitada por 
sua pequena espessura e alta heterogeneidade litológica, tendo o seu potencial de explotação viabilizado apenas na presença e continuidade espacial de pacotes arenosos. Assim, a granulometria pode ser utilizada vantajosamente para entender melhor as propriedades hidráulicas das unidades hidrogeológicas, já que oferecem dados para estudos detalhados e de maior resolução.

\section{REFERÊNCIAS}

ASSOCIAÇÃO BRASILEIRA DE NORMAS TÉCNICAS (ABNT). Solo: análise granulométrica, (MB 32) NBR 7181. Rio de Janeiro, 13 p. 1984.

ASTM - AMERICAN SOCIETY FOR TESTING AND MATERIALS. Standard method for specific gravity of soils. Philadelphia, PA: ASTM D 854, 1998.

CHANG, H. K.; CARNIER NETO, D. Winslug. INPI Registro \# 03239-1 (11/07/2000). Revista da Propriedade Industrial, Seção I, n. 2302, p. 115, 2015.

CHAPUIS, R. P. et al. Evaluating the hydraulic conductivity at three different scales within an unconfined sand aquifer at Lachenaie, Quebec. Canadian Geotechnical Journal, v. 42, n. 4, p. 1212-1220, 2005.

CHEONG, J. Y. et al. Estimating hydraulic conductivity using grain-size analyses, aquifer tests, and numerical modeling in a riverside alluvial system in South Korea. Hydrogeology Journal, v. 16, n. 6, p. 1129-1143, 2008.

COTTAS, L. R. Estudos geológico- geotécnicos aplicados ao planejamento urbano de Rio Claro - SP. São Paulo, SP. 171p., 2V. (Tese de Doutoramento - Instituto de Geociências/ USP). 1983.

FERREIRA, S. R. Análise pedoestratigráfica das formações Rio Claro e Piraçununga, no Centro-Leste do Estado de São Paulo. 2005. xiii, 157 f. Tese (doutorado) - Universidade Estadual Paulista, Instituto de Geociências e Ciências Exatas, 2005.

FERREIRA, S. R.; CAETANO-CHANG, M. R. Datação das formações Rio Claro e Piraçununga por termoluminescência. R. Esc. Minas, v. 61, n. 2, p. 129-134, jun. 2008.

FETTER, C. W. Applied hydrogeology. 4. ed. New Jersey: Prentice Hall, 2001. 598 p.

GONÇALVES, R. D.; CHANG, H. K. Modelo hidrogeológico do Sistema Aquífero Urucuia na Bacia do Rio Grande (BA). Geociências, v. 36, n. 2, p. 205-220, 2017.

HVORSLEV, M. J. Time lag and soil permeability in ground water observations. Bul. Army Corps of Engineers Waterway Experimentation Station, U. S. n. 36, 1951.

KRUMBEIN, W. C.; MONK, G. D. Permeability as a function of the size parameters of unconsolidated sands. American Institute of Mining and Metallurgical Engineers, v. 1492, p. $1-11,1942$.

MELO, M. S. DE; COIMBRA, A. M.; CUCHIERATO, G. Fácies sedimentares da formação Rio 
Claro, Neocenozóico da depressão periférica paulista. Revista do Instituto Geológico, v. 18, n. 1/2, p. 49-63, 1997.

OLIVA, A.; KIANG, C. H.; CAETANO-CHANG, M. R. Determinação da condutividade hidráulica da Formação Rio Claro: análise comparativa através de análise granulométrica e ensaios com permeâmetro guelph e testes de slug. Águas Subterrâneas, v. 19, n. 2, p. 1-17, 2005.

OLIVA, A. Estudo hidrofaciológico do aqüífero Rio Claro no município de Rio Claro - SP.

(Tese de Doutorado), Universidade Estadual Paulista, Instituto de Geociências e Ciências Exatas, Rio Claro (SP). 2006. 196 p.,

PAULA E SILVA, F. DE; CHANG, H. K. Interferências da atividade minerária argileira nos recursos hídricos da bacia do Ribeirão Santa Gertrudes / SP. São Paulo, 2017.

ROSAS, J. et al. Determination of Hydraulic Conductivity from Grain-Size Distribution for Different Depositional Environments. Groundwater, v. 52, n. 3, p. 399-413, maio 2014.

SHEPHERD, R. G. Correlations of Permeability and Grain Size. Ground Water, v. 27, n. 5, p. 633-638, set. 1989.

STRADIOTO, M. R.; CHANG, H. K. Caracterização Hidroquímca e Isotópica das Águas Superficiais, Pluviais e Subterrâneas do Município de Rio Claro-SP. In: XVI CONGRESSO BRASILEIRO DE ÁGUAS SUBTERRÂNEAS E XVII ENCONTRO NACIONAL DE PERFURADORES DE POÇOS, 16, 2010. Anais..., 2010.

TERAMOTO, E. H. et al. Modelo Empírico para estimativa de Condutividade Hidráulica a partir de dados granulométricos. Geotecnia, n. 119, p. 41-53, 2010.

UNITED STATES ARMY CORPS OF ENGINEERS (USACE). Hudson-raritan estuary, liberty state park ecosystem restoration: environmental impact statement, v. 3, 2005. 23 p. 